\section{NTP Process for Preparation of the Report on Carcinogens}

The Report on Carcinogens ( $\mathrm{RoC})$ is a Congressionally mandated, science-based public health document that identifies and discusses agents, substances, mixtures, or exposure circumstances (collectively referred to as "substances") that pose a cancer hazard for persons in the United States. Substances are listed in the report as either known or reasonably anticipated to be human carcinogens. The RoC has a substance profile for each listing that includes a description of the substance, its uses and potential sources of exposure, a summary of the scientific information considered key to its listing, and applicable, current federal regulations and guidelines to limit exposure. Each edition of the report is cumulative and includes newly reviewed substances and those previously listed. The National Toxicology Program (NTP $)^{1}$ prepares the RoC on behalf of the Secretary of Health and Human Services.

The process for preparation of the RoC has four parts: (1) select substances for evaluation, (2) prepare draft RoC monographs for selected substances, (3) convene peer review and finalize RoC monographs, and (4) submit new listings for approval and publish the RoC. Each part is described briefly below and depicted in the schematic.

\section{Step 1: Select Substances for Evaluation}

NTP invites nominations of substances for review for possible listing in the RoC from anyone in the public or private sector. Nomina-

${ }^{1}$ NTP is a federal interagency program, headquartered at the National Institute of Environmental Health Sciences, part of the National Institutes of Health, whose goal is to safeguard the public by identifying substances in the environment that may affect human health. For more information about NTP and its programs, visit http://ntp.niehs.nih.gov. tions may be chemical, physical, or biological substances or classes of substances. A nomination may seek to list a new substance in the RoC, reclassify the listing status of a substance already listed, or remove a listed substance. Nominations may be submitted by mail or email $^{2}$ or online at http://ntp.niehs.nih.gov/go/152.

For each nomination, NTP conducts scoping and problem formulation activities to determine whether adequate information on exposure and carcinogenicity exists to justify evaluation for the RoC. These activities may include preliminary literature searches to identify publicly available studies, public solicitation of information on exposure or ongoing studies of carcinogenicity through the Federal Register and NTP publications, and consultation with external technical advisors and/or NTP partner agencies. ${ }^{3}$ Public comments on nominations are posted to the NTP website. ${ }^{4}$ NTP uses these inputs to identify substances for evaluation for the RoC.

For each substance proposed for RoC evaluation, NTP prepares a draft concept document, which is informed by the scoping and problem formulation activities. A "concept" is a brief document that

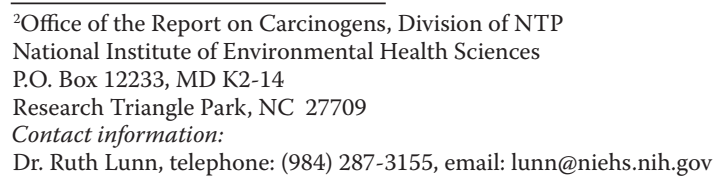

${ }^{3}$ Interagency review is invited from agencies represented on the NTP Executive Committee, including the Consumer Product Safety Commission, Department of Defense, Environmental Protection Agency, Food and Drug Administration, National Cancer Institute, National Center for Environmental Health/Agency for Toxic Substances and Disease Registry, National Institute of Environmental Health Sciences, National Institute for Occupational Safety and Health, and Occupational Safety and Health Administration. ${ }^{4}$ Public comments received are available at http://ntp.niehs.nih.gov/pubhealth/roc/noms/ index.html.

\title{
Process for the Preparation of the RoC
}

\section{Select substances for evaluation}

Invite nominations
Conduct scoping and
problem formulation
activities
$\begin{aligned} & \text { Scientific and/or } \\ & \text { public input } \\ & \text { as needed }\end{aligned}$
Develop draft concepts
Public comment
NTP BSC* review
(public meeting \&
comment)
NTP Director

Finalize concepts and select substances for review

\section{Prepare draft RoC monographs}

\section{Develop protocol as needed}

Scientific and/or public input as needed

Develop draft RoC monograph

Scientific and/or public input as needed

Interagency review of NTP listing recommendation

\section{Convene peer review and finalize RoC monographs}

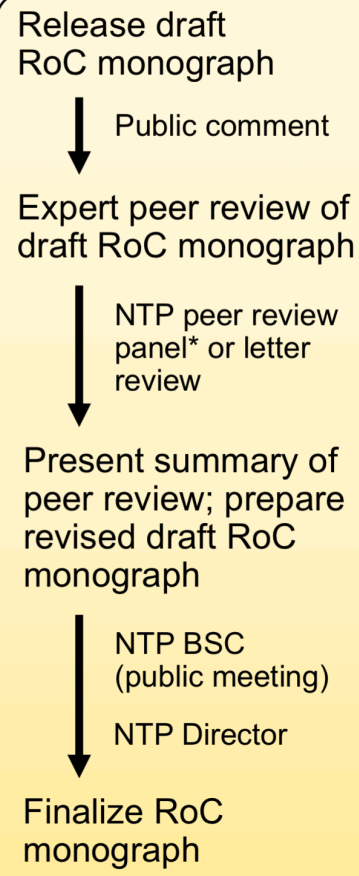

Release draft RoC monograph $\downarrow$ Public comment Expert peer review of draft RoC monograph

NTP peer review panel $^{\star}$ or letter review

Present summary of peer review; prepare revised draft RoC monograph

NTP BSC (public meeting) NTP Director

Finalize RoC monograph

4. Submit for approval and publish RoC

Submit recommended listing status of newly reviewed substances

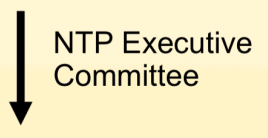

Secretary, HHS reviews and approves

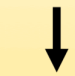

Publish and release RoC 
presents the rationale, public health significance, and objective of the evaluation. It may also discuss information on exposure, concern for potential carcinogenicity, key scientific issues or questions, and the general approach for preparing the RoC monograph (see below). NTP announces availability of draft concept documents on substance(s) proposed for evaluation and solicits public comments on them through the Federal Register and NTP publications.

NTP presents the draft concept document for a substance to the NTP Board of Scientific Counselors (BSC) $)^{5}$ at a public meeting with opportunity for public comment. The BSC is asked to comment on the merit of the proposed evaluation and provide input on the draft concept document. NTP considers the BSC and public comments, and the NTP Director makes the final determination whether to select the substance for formal $\mathrm{RoC}$ evaluation. Concept documents for selected substances are finalized based upon those comments. For each selected substance, NTP sets up a webpage on the NTP website to post materials related to its RoC evaluation.

\section{Step 2: Prepare Draft RoC Monographs for Selected Substances}

The cancer hazard evaluation of a selected substance is captured in an RoC monograph. The RoC monograph typically presents information on human exposure to the substance, especially U.S. exposure, and an assessment of the evidence from cancer studies in humans and experimental animals, mechanisms of carcinogenicity, and other data (such as absorption, distribution, metabolism, and excretion) relevant for evaluating the substance's potential carcinogenicity. The draft RoC monograph presents NTP's preliminary conclusions regarding the level of evidence for carcinogenicity from studies in humans or experimental animals and its preliminary RoC listing recommendation. These conclusions are reached by applying the RoC listing criteria $^{6}$ to the cancer assessment. In most instances, the RoC monograph includes the draft substance profile as a separate section; however, in some instances, the monograph represents the RoC substance profile.

The specific approach for preparing the draft RoC monograph for a substance is guided by the nature, extent, and complexity of the scientific information and tailored to address the key scientific issues or questions for evaluating whether the substance is a potential cancer hazard. The Handbook for the Preparation of Report on Carcinogens Monographs ${ }^{7}$ provides general instructions on the process. Typically, the first step in the process is to develop a protocol, which adapts the general instructions in the handbook to address the key scientific issues or questions related to the particular substance. The protocol is posted to the substance's webpage.

All public comments received during preparation of the draft $\mathrm{RoC}$ monograph for a substance become part of the public record, are

\footnotetext{
${ }^{5}$ The NTP Board of Scientific Counselors (BSC) is a federally chartered advisory committee whose members are appointed by the Secretary of Health and Human Services. The BSC provides advice to the NTP Director on matters relating to scientific program content and evaluates the scientific merit of the NTP's intramural and collaborative programs. Information about the BSC is available at http://ntp.niehs.nih.gov/go/164. ${ }^{6} \mathrm{RoC}$ listing criteria are the standards against which the scientific evidence for carcinogenicity is evaluated to determine whether a substance should be listed in the RoC and, if so, whether as known to be a human carcinogen or reasonably anticipated to be a human carcinogen. The criteria are available at http://ntp.niehs.nih.gov/go/15209.

${ }^{7}$ The handbook is available at http://ntp.niehs.nih.gov/go/rochandbook.
}

posted to the substance's webpage, and are considered by NTP and any technical advisors during subsequent steps in the RoC evaluation process. NTP also seeks input on the preliminary RoC listing recommendation for the substance from its partner agencies and then completes the draft RoC monograph, with consideration of all inputs to its development.

\section{Step 3: Convene Peer Review and Finalize RoC Monographs}

NTP publishes a Federal Register notice announcing peer review and availability of the draft RoC monograph and inviting written public comment. NTP follows Department of Health and Human Services peer-review policies, ${ }^{8}$ which were developed in accordance with the Office of Management and Budget's Final Information Quality Bulletin for Peer Review, ${ }^{9}$ to select and carry out an appropriate peer-review mechanism (e.g., by panel or letter). When peer review occurs by a panel in a public forum, NTP invites the public to attend the meeting and provide oral comments.

Peer reviewers are charged to (1) comment on whether the draft RoC monograph is technically correct, clearly stated, and objectively presented, (2) provide opinion on whether there is currently or was in the past significant human exposure to the substance for persons residing in the United States, and (3) vote on whether the scientific evidence supports the level-of-evidence conclusions regarding carcinogenicity and NTP's preliminary policy decision on the substance's listing status in the RoC. A report on the peer review is prepared and posted to the substance's webpage.

Based upon the peer-review comments, NTP prepares a revised draft RoC monograph. At a public meeting, NTP provides the NTP Board of Scientific Counselors with information about the peer review. Following the meeting, NTP finalizes the RoC monograph for the substance and posts it to the substance's webpage. The final monograph represents NTP's policy recommendation on the substance's listing status in the RoC.

\section{Step 4: Submit New Listings for Approval and Publish the RoC}

NTP submits the draft substance profiles for newly reviewed substances with their recommended listing status to the NTP Executive Committee $^{10}$ for consultation and then to the Secretary of Health and Human Services for review and approval. Upon approval by the Secretary, the substance profiles for newly reviewed substances are added to the next edition of the $\mathrm{RoC}$, and the $\mathrm{RoC}$ is prepared in electronic format, transmitted to Congress, and published on the NTP website for the public. Periodically, NTP publishes the RoC in both printed and electronic formats, depending upon demand for the printed version.

NTP publishes a notice in the Federal Register and NTP publications announcing the listing outcome for each substance that underwent formal evaluation for the RoC and availability of the next edition of the RoC.

\footnotetext{
${ }^{8}$ The policies are available at https://aspe.hhs.gov/hhs-information-quality-peer-review. ${ }^{9}$ The bulletin is available at https://www.whitehouse.gov/sites/default/files/omb/assets/ omb/fedreg/2005/011405_peer.pdf.

${ }^{10} \mathrm{NTP}$ Executive Committee, see footnote 3.
} 